\title{
A MICROMACHINED PLANAR PATCH-CLAMP CHIP WITH INTEGRATED MICROFLUIDICS
}

\author{
Brian Matthews and Jack W. Judy \\ Department of Electrical Engineering, University of California, Los Angeles \\ Los Angeles, California, 90095
}

\begin{abstract}
We have designed, fabricated, integrated, and tested microfabricated planar patch-clamp substrates and poly(dimethylsiloxane) (PDMS) microfluidic components suitable for use in an automated planar patch-clamp dose-response measurement system. Substrates with cell-patch-site aperture diameters ranging from $300 \mathrm{~nm}$ to $12 \mu \mathrm{m}$ were produced using standard MEMS fabrication techniques. The resistance of the cellpatch sites and substrate capacitance were measured using impedance spectroscopy. The resistance of the cell-patch sites ranged from $200 \mathrm{k} \Omega$ to $47 \mathrm{M} \Omega$ for apertures ranging from $12 \mu \mathrm{m}$ to $750 \mathrm{~nm}$ respectively. The substrate capacitance was $17.2 \mathrm{pF}$ per $\mathrm{mm}^{2}$ of fluid contact area for substrates with a $2-\mu \mathrm{m}$-thick layer of silicon dioxide. In addition, testing shows that the fluidic components are appropriate for driving human embryonic kidney cells (HEK 293) to patch apertures, for trapping cells on patch apertures, and for rapidly exchanging the extracellular fluid environment.
\end{abstract}

\section{INTRODUCTION}

$I^{2}$ ON-CHANNEL proteins, which are embedded in cell membranes, control the flow of ions through the membrane and are gated by chemical, electrical, or mechanical stimuli. The ionic currents generated from the gating of ion-channels control many cellular processes, such as cell-signaling pathways and have been studied extensively using the patch-clamp technique. The patchclamp technique is a powerful tool for the investigation of many electrophysiological cell properties, such as the characterization of the kinetics and steady-state effects of toxins, chemical

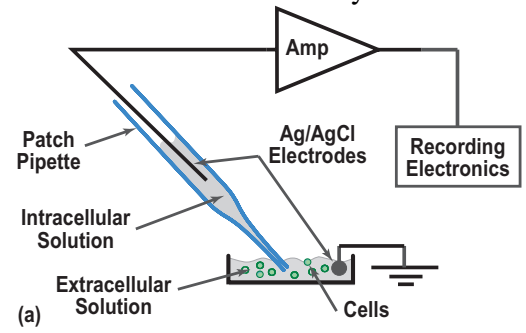

(b)
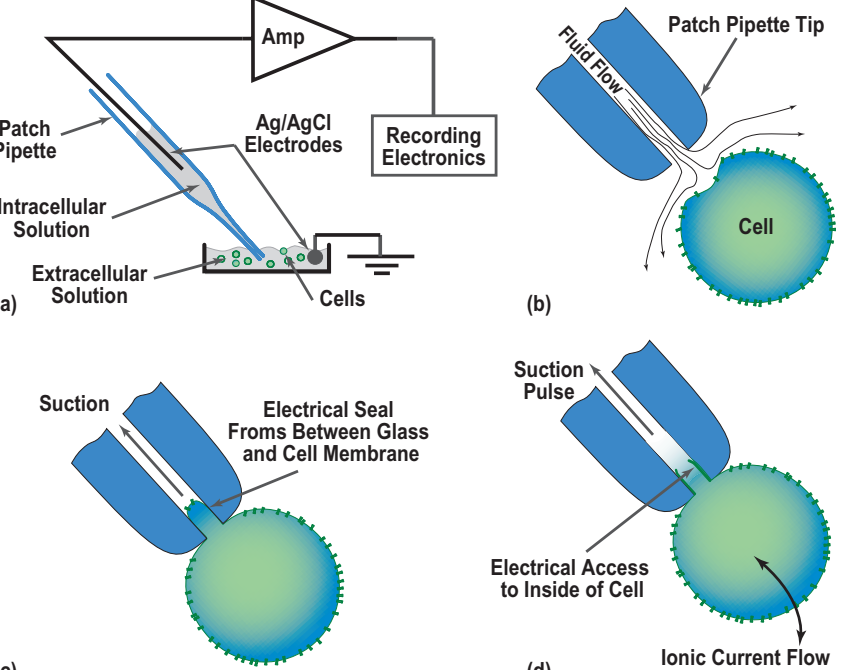

(c)

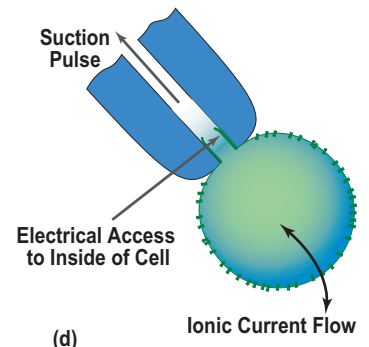

Fig. 1. (a) Schematic representation of a traditional patch-clamp system using a pulled-glass pipette. (b) The pipette is positioned close to a cell with positive pressure to prevent contaminating the tip with debris from the solution. (c) Suction is applied to the pipette and a highly resistive electrical seal (i.e., gigaohm seal) is formed at the interface between the glass and cell membrane. (d) A suction pulse is applied, and the cell membrane inside the pipette lumen is ruptured to provide direct electrical access to the inside of cell.
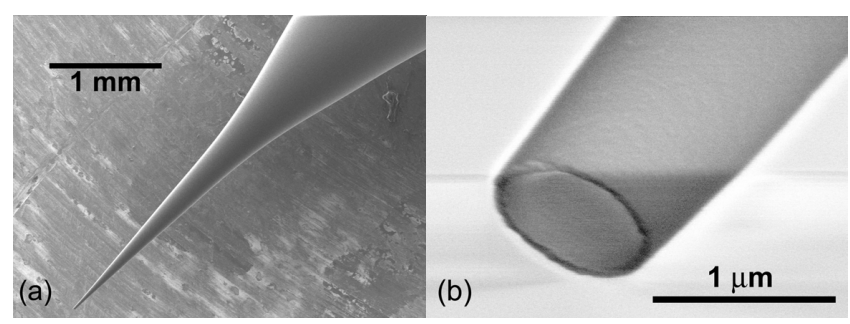

Fig. 2. (a) SEM of an uncoated traditional patch-clamp pipette and (b) close-up of the pipette tip.

agonists, antagonists, and drugs in ion channels, transporters, and pumps [1]. Specifically, the patch-clamp technique enables the measurement of ionic current flowing through the cellular membrane by creating isolated electrical connections to the inside and outside of a cell membrane (Fig. 1). In the traditional patchclamp technique, an approximately 1.5 -mm-diameter glass capillary is heated and pulled apart to form a pipette with a tip diameter of $500 \mathrm{~nm}$ to $10 \mu \mathrm{m}$, depending on the intended application (Fig. 2) [2]. The pipette is filled with a suitable solution and electrically connected to electronics for amplification and recording, via a $\mathrm{Ag} / \mathrm{AgCl}$ non-polarizable electrode. The pipette is then used to electrically isolate the intracellular and extracellular sides of a small patch of membrane. By forming a seal to the membrane and achieving electrical isolation on the order of gigaohms, the pipette is able to record currents passing through the membrane to a ground electrode (Fig. 1a). Using the configuration shown in Fig. 1, it is possible to investigate the electrical and chemical dependencies of ion channels. Although the traditional equipment used to perform the patch-clamp technique has been very successful, conventional pipettes are not easily adapted to all the applications for which the patch-clamp technique would be useful. Examples of electrophysiological applications that would benefit from uniquely tailored patch-clamp systems include measuring dose-response curves for single cells, high-throughput screening, applications needing optical access to the cell (e.g., confocal and florescence microscopy), and applications needing rapid intracellular perfusion.

\section{MOTIVATION}

With the advent of micromachining and microelectromechanical systems (MEMS) technologies, specifically engineered patch-clamp systems can be realized. Microfabricated patch-clamp systems hold the promise of parallelism, microfluidic manipulation, uniformity, optical access, and automation that is currently impossible to obtain with traditional pulled-glass capillary systems. Although microfabricated structures have been used to patch to cells with gigaohm seals in academic and commercial laboratories, these devices do not patch with high reliability, have not been integrated into miniaturized systems, or have solely targeted high-throughput screening [4-13]. The ultimate goal of this work is to develop a set of microfabrication processes that are flexible enough to address a number of application-specific patch-clamp configurations and to create a microfluidic system capable of performing complex electrophysiological experiments on individual cells. 


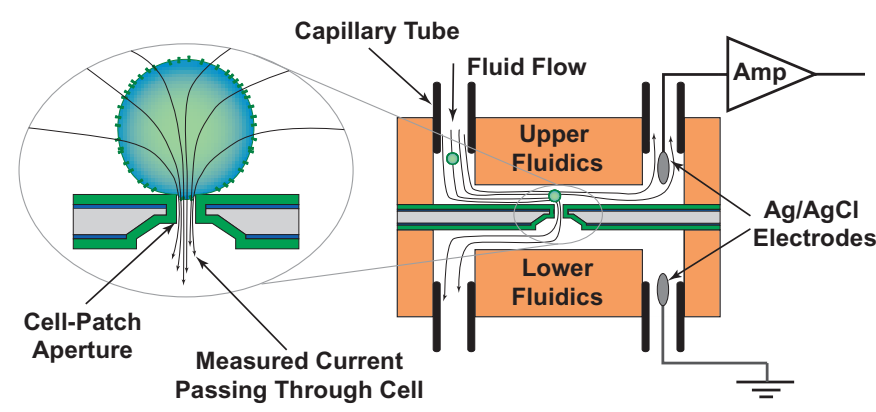

Fig. 3. Schematic diagram of a planar patch-clamp dose-response measurement system.

\section{DESIGN}

Our initial effort will be to microfabricate a system capable of automating the measurement of single-cell dose-response curves in stablely transfected human embryonic kidney (HEK), Chinese hamster ovary (CHO), and other cell lines. Dose-response measurements are used to evaluate the effect of chemical concentration on ion channels. Therefore, it is necessary for a dose-response system to be able to apply different concentrations of a chemical in a precise and repeatable way. Such an automated system will necessarily include the base components needed for most specialized patch-clamp experiments. Components such as: a suitable cell-patch site, $\mathrm{Ag} / \mathrm{AgCl}$ electrodes, microfluidic components to precisely control intracellular and extracellular solutions, good optical access, and system-wide mechanisms to control the recording process.

The optimal diameter for cell-patch sites dependings on the particular application and the size of cell being investigated. Cell lines such as HEK, $\mathrm{CHO}$, and others typically have cell sizes ranging from $7 \mu \mathrm{m}$ to $10 \mu \mathrm{m}$. Acceptable cell-patch-site diameters, when working with cells of this size, are from $500 \mathrm{~nm}$ to $2.5 \mu \mathrm{m}$. Although, the target aperture size for this project was $1 \mu \mathrm{m}$, the design of the process allows for adjustments in this diameter to be made without changing the mask set. Silicon dioxide was chosen as the surface material for the cell-patch sites in an attempt to simulate traditional pulled-glass pipettes.

The initial design of the microfluidic components was made intentionally simple. The design of the topside microfluidic system includes eight ports that provide direct fluid access to a patched cell: a cell-injection port, six solution-exchange ports, and a waste port (Fig. 4). For this initial microfluidic system, the front-side channels are $30-\mu \mathrm{m}$ wide and $30-\mu \mathrm{m}$ deep.

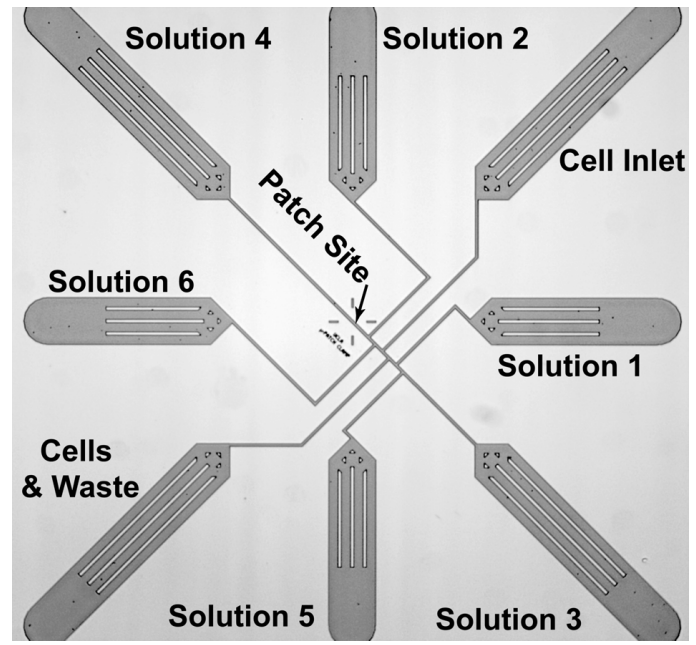

Fig. 4. Overview of the eight-port microfluidic system.
When used for conventional dose-response measurements, the six solution-exchange ports can be configured to provide a patched cell with six different premixed concentrations of a chemical. In this configuration the six predefined chemical concentrations are sequentially applied to a cell in a continuous fashion and for a period of time necessary for the cell response to reach equilibrium.

An alternative method of delivering chemical stimuli to a patched cell is with the use of sample plugs. A sample plug can be formed by a pair of offset solution-exchange channels crossing the cell-injection channel. This microfluidic configuration is similar to the sample-preparation methods used in capillary electrophoresis systems [14]. The sample plugs can be used to study the kinetics of ion-channel activation and deactivation. Currently backside fluidic connections consist of a simple two-port structure machined into the polycarbonate test fixture that connects to the backside of the cell-patch aperture

Similar to traditional patch-clamp pipettes, we anticipate that planar patch-clamp chips cannot be reused once a recording is made. Because of the single-use nature of the system, it is necessary for the planar system to have an easy method for exchanging chips. In order to facilitate quick macrofluidic connections, the microfluidics are fabricated with a thick layer of PDMS containing integrated access ports capable of accepting capillary tubing without the need for glue. The completed, assembled and plumbed system is shown in Fig. 5.

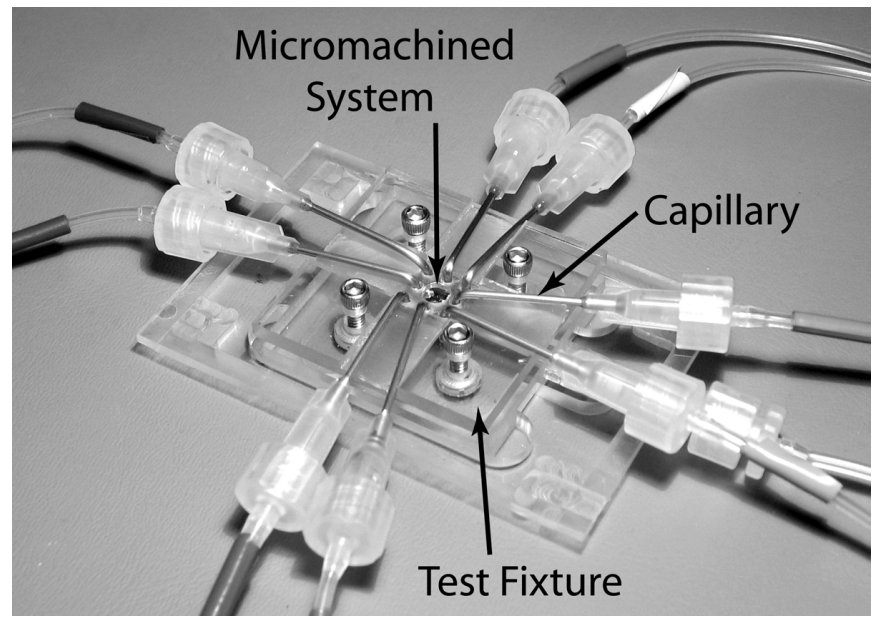

Fig. 5. Photograph of the macroscopic test fixture with eight capillaries connecting to the micromachined planar patch-clamp system.

\section{FABRICATION}

\section{A. Silicon-Based Patch-Clamp Substrates}

The fabrication process of the substrates containing the cellpatch sites is a two-mask process and was carried out at the UCLA Nanoelectronic Research Facility. A schematic cross section of a substrate is given in Fig. 6a. The process begins with a 100-mmdiameter p-doped $\{100\}$ silicon wafer. (Silicon Quest International).

The wafers are cleaned in 5:1 $\mathrm{H}_{2} \mathrm{SO}_{4}: \mathrm{H}_{2} \mathrm{O}_{2}$ (piranha) and inserted into a low-temperature low-pressure chemical-vapordeposition (LPCVD) furnace at $790{ }^{\circ} \mathrm{C}$ and $33 \mathrm{~Pa}$ (0.25 torr) with $40 \mathrm{sccm}$ of $\mathrm{SiH}_{2} \mathrm{Cl}_{2}$ and $108 \mathrm{sccm}$ of $\mathrm{NH}_{3}$ in order to deposit 200 $\mathrm{nm}$ of silicon nitride $\left(\mathrm{Si}_{3} \mathrm{~N}_{4}\right)$ (Tystar Titan II). The first photolithography step is then performed on the backside of the wafers using AZ5214EIR photoresist (Clariant Ltd.) using a standard process provided by the resist manufacturer [13]. 


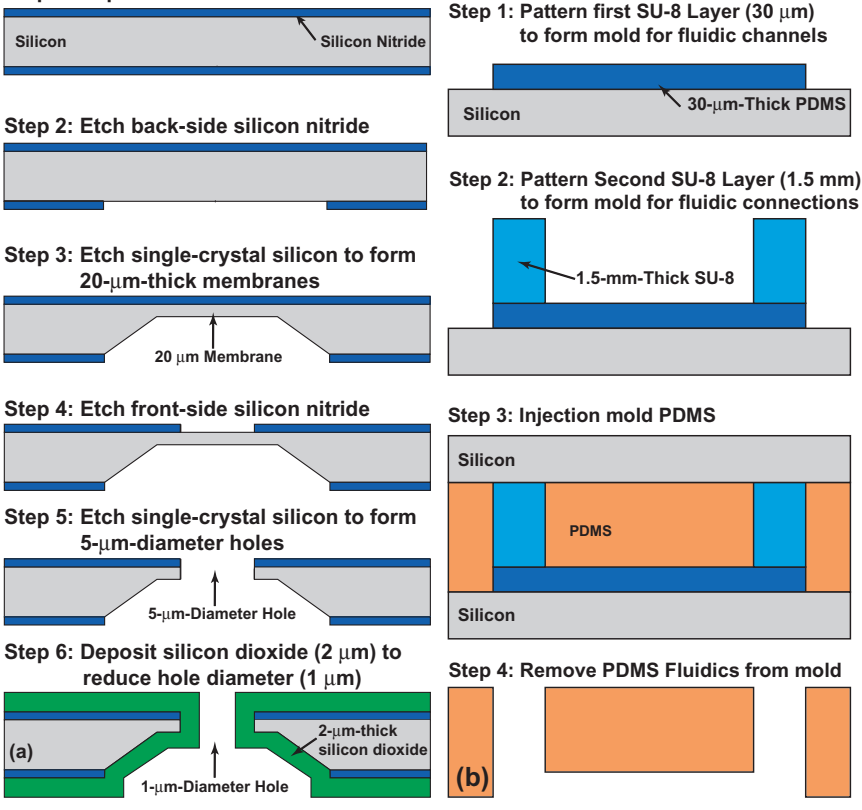

Fig. 6. (a) Cell-patch-site fabrication process and (b) microfluidic fabrication process.

Openings in the backside silicon nitride layer are then etched in a reactive ion etcher (RIE) at $13 \mathrm{~Pa}$ ( 0.1 torr), $200 \mathrm{~W}$, and 5:1 $\mathrm{CF}_{4}: \mathrm{O}_{2}$ for $3 \mathrm{~min}$ (Techniques Fluorine RIE 800). The wafers are then striped of photoresist and submerged in a $30 \%$ solution of $\mathrm{KOH}$ to etch the single-crystal silicon to form membranes as defined by the backside silicon-nitride openings. As there is nothing to stop the $\mathrm{KOH}$ from completely etching through the wafer, it is necessary to carefully monitor the etch rate and remove the wafers at the appropriate time.

The wafers are then cleaned and the second photolithography step is performed, again using AZ5214EIR, to define the cell-patch sites. The front-side silicon nitride is then etched using the same RIE process as the backside silicon nitride. A deep reactive ion etcher (DRIE), using a standard Bosch59 process, is then used to create a hole through the membrane that forms the cell-patch site (PlasmaTherm SLR Series). Once the hole is formed, the wafers are cleaned in piranha for $20 \mathrm{~min}$ and then further cleaned in an oxygen plasma at $150{ }^{\circ} \mathrm{C}, 66 \mathrm{~Pa}(0.5$ torr), and $200 \mathrm{~W}$ for $30 \mathrm{~min}$ (Tegal Plasmaline 515). This cleaning is necessary to remove the fluorine-based polymer deposited in the DIRE process.

The wafers are then cleaned again in piranha for $10 \mathrm{~min}$ and placed into either an LPCVD furnace or a thermal-oxidation furnace to deposit a layer of silicon dioxide $\left(\mathrm{SiO}_{2}\right)$ (Tystar Titan II). The 2- $\mu \mathrm{m}$-thick layer of $\mathrm{LPCVD} \mathrm{SiO}_{2}$ is deposited at $450{ }^{\circ} \mathrm{C}$ and $33 \mathrm{~Pa}\left(0.25\right.$ torr) with $43 \mathrm{sccm}$ of $\mathrm{SiH}_{4}$ and $60 \mathrm{sccm}$ of $\mathrm{O}_{2}$. For wafers receiving thermal oxidation, the silicon nitride was removed from both sides of the wafer using the RIE process described above. The 2- $\mu \mathrm{m}$-thick layer of thermal $\mathrm{SiO}_{2}$ is grown at $1100^{\circ} \mathrm{C}$ for $8.5 \mathrm{hr}$ in the presence of saturated water vapor. Because the oxide deposition techniques are conformal, the deposited film will coat the sidewalls of the hole, thereby reducing its diameter. Silicon dioxide, in addition to reducing the diameter of the hole, will form a surface onto which cells can form a tight seal. It is therefore possible to fabricate cell-patch sites with a wide range of diameters and with diameters smaller than the lithographic capabilities of our optical mask aligner. The wafers are then cleaved into $1-\mathrm{cm}^{2}$ dice in preparation for testing. After cleaving, the backsides of several substrates are bonded to $1-\mathrm{mm}$ thick poly(dimethylsiloxane) (PDMS) gaskets with $1-\mathrm{mm}^{2}$ openings in order to investigate the effect of fluid contact area on capacitance.

\section{B. Microfluidics}

The microfluidic components of the system were fabricated by molding PDMS onto a two-level SU-8 mold master [15]. Schematic cross sections of the process are given in Fig. $6 \mathrm{~b}$. The first layer of SU-8 is then deposited on a 100 -mm-diameter silicon wafer using a standard process provided by the resist manufacturer [16]. This first layer is made from 30- $\mu$ m-thick SU-8 2025 and forms the mold for the microfluidic channels. The second layer of SU-8 is fabricated from a 2-mm-thick layer of SU-8 2100 and forms the macroscale fluid-access ports. The fabrication process for the second SU-8 layer is adapted from a process developed by Lin et. al. and is described below [17].

Because a 2-mm-thick layer of SU-8 will flow off the edges of the wafer when brought above the glass transition temperature, a 2-mm tall casting barrier is first glued around the periphery of the silicon wafer containing the first SU-8 layer. The wafer is then dehydrated at $150{ }^{\circ} \mathrm{C}$ for $10 \mathrm{~min}$. A pre-measured amount of SU-8 2100 is then poured into the casting barrier to form a 2 -mm-thick layer of SU-8. The pre-exposure bake is then performed at $120^{\circ} \mathrm{C}$ for $8 \mathrm{hr}$ without initial ramping of the hotplate. At the end of the 8hr softbake time, the hotplate was cooled to $60^{\circ} \mathrm{C}$ and held for $5 \mathrm{~min}$. The temperature is then ramped down to $50^{\circ} \mathrm{C}$ at a rate of $20{ }^{\circ} \mathrm{C} \cdot \mathrm{hr}^{-1}$. The wafer is then exposed with a total exposure dose of $5.6 \mathrm{~J} \cdot \mathrm{cm}^{-2}$ (Karl Suss MA6). The post exposure bake is performed at $65^{\circ} \mathrm{C}$ for $10 \mathrm{~min}$ and then $100{ }^{\circ} \mathrm{C}$ for $10 \mathrm{~min}$ starting from room temperature with a ramp rate of $150^{\circ} \mathrm{C} \cdot \mathrm{hr}^{-1}$. The wafer is then submerged in SU-8 developer until the features are completely developed, a process that typically takes 1 to $2 \mathrm{hr}$ (SU-8 Developer, Micro Chem). After being fully developed, rinsed, and dried, a flood exposure of $5.6 \mathrm{~J} \cdot \mathrm{cm}^{-2}$ and hard bake of $150{ }^{\circ} \mathrm{C}$ for $10 \mathrm{~min}$ are then performed to further crosslink the SU-8 and drive off residual solvent.

Once complete, the SU-8 mold master is used to create the PDMS microfluidic components. In order to ensure that the macroscale fluidic access ports remain unoccluded during the casting process, a blank silicon wafer is then clamped on top of the SU-8 mold master and PDMS is then injected into the interstitial space between the two wafers. The PDMS is cured in an oven at $65^{\circ} \mathrm{C}$ for $1 \mathrm{hr}$ (Blue M). Subsequent to curing the PDMS is removed from the mold master and cut to form $1-\mathrm{cm}$ dice. The macroscale fluid-access ports provide an easily operated and robust fluidic connection technology that seals to capillary tubing without glue.

Patch-clamp substrates and PDMS microfluidics were aligned and bonded using a mask aligner/bonder (Karl Suss MA-6). Prior to bonding the patch-clamp substrates and PDMS microfluidics are exposed to an oxygen plasma (100 W for $1 \mathrm{~min})$ to modify the PDMS surface to form a bond with adhesion strength greater than that of the bulk PDMS [18].

\section{EXPERIMENTAL RESULTS}

\section{A. Physical Properties}

The physical properties of the cell-patch sites were investigated using a scanning electron microscope (SEM) on uncoated samples (Hitachi S4700). The micrograph in Fig. 7 shows the smoothness and cleanliness of a cell-patch site prior to silicon dioxide deposition. It was found that if the cell-patch site had any residual polymer or other debris from the DIRE, the silicon dioxide layer would deposit in a non-uniform manner. The result is an out-of-round aperture unsuitable for seal formation. The micrograph in Fig. 8 is an example of typical cell-patch site. 


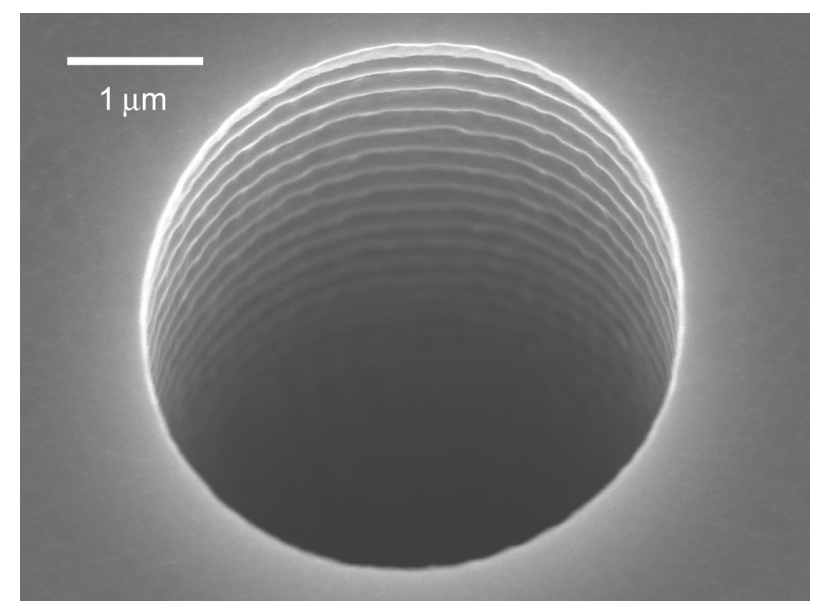

Fig. 7. Cell-patch site before oxide deposition or growth.

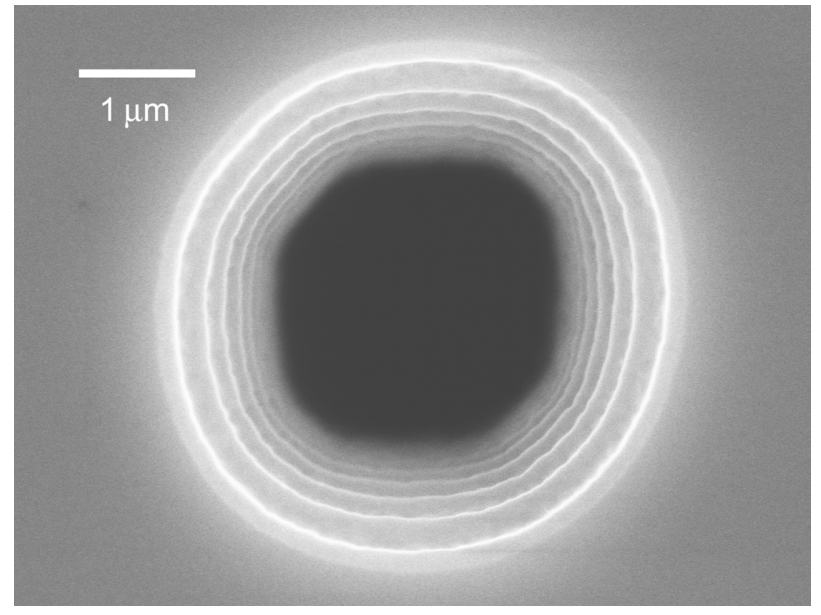

Fig. 8. (b) An example of a 2.5- $\mu$-diameter cell-patch site made of thermal $\mathrm{SiO}_{2}$.

\section{B. Electrical Properties}

The small signals generated by ion channels in patch-clamp experiments can be obscured by noise from the following general categories: biological sources, experimental apparatus, and the interface with the experimental apparatus to the biology. Contributions to the general noise categories are made by many sources in the experiment, such as the amplifier, cell membrane, glass-membrane seal, pipette holder, pipette, and interactions between all of these sources [19]. Because the proposed devices are replacing the pipette holder and pipette used in traditional patch-clamp experiments, the noise sources associated with these replaced components must be well understood. In conventional patch-clamp systems the pipette holder and pipette contribute to several noise terms through lossy dielectrics, capacitance added to the amplifier input by the pipette holder and pipette, distributed RC noise, and noise arising from the resistance of the pipette in series with the cell membrane. As a general rule, in attempting to perform low-noise single-channel recordings, it is advantageous to reduce both the series resistance and capacitance traditionally associated with the pipette. As an example, reducing the capacitance of the pipette will reduce dielectric noise that results from the thermal fluctuations in lossy dielectrics.

In order to extract the series resistance and capacitance of our substrates, a potentiostat was used to measure their impedance spectra (EG\&G Instruments 283 and Solatron SI 1260). To perform measurements the samples were place in a testing apparatus and electrically isolated $100-\mathrm{mM}-\mathrm{KCl}$ electrolyte solutions were placed in contact with the frontside and backside of the substrate. $\mathrm{An} \mathrm{Ag} / \mathrm{AgCl}$ non-polarizable electrode was then placed into each $\mathrm{KCl}$ electrolyte solution. The $\mathrm{Ag} / \mathrm{AgCl}$ electrodes were connected to the potentiostat and the impedance spectra were measured using a $10-\mathrm{mV}$ excitation voltage with a $1 \mathrm{~Hz}$ to $10^{5} \mathrm{~Hz}$ frequency range. The equivalent circuit, shown in Fig. 9a, was used to model the impedance spectra. The series resistance of the aperture and the substrate capacitance in the circuit model were fitted to the measured impedance spectra.
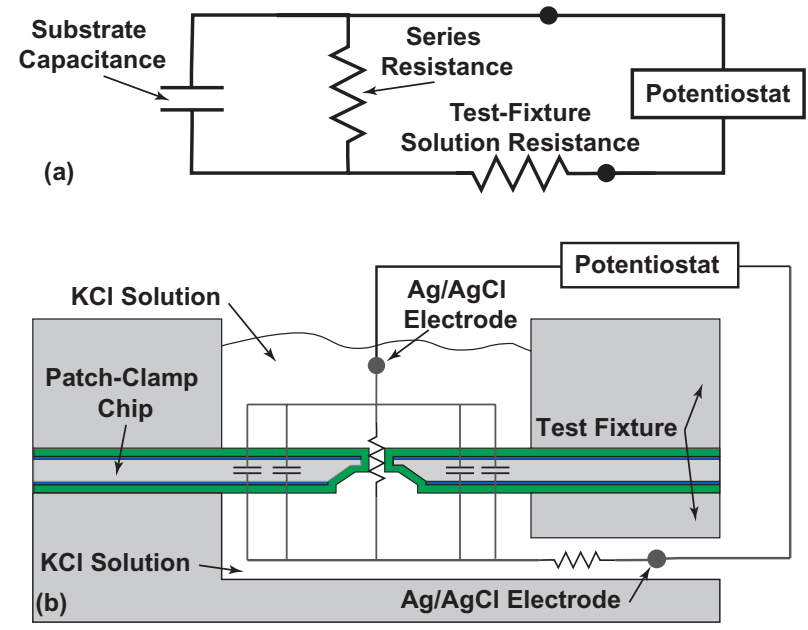

Fig. 9. (a) Equivalent-circuit model of cell-patch-site substrates. (b) Schematic diagram showing physical locations of parameters in the equivalent circuit model.

Simple resistance and capacitance models were developed for the substrates and are illustrated in Fig. 9b. The overall system resistance is dominated by the conductance of the cell-patch-site apertures because of their small dimensions relative to the rest of the conducting path. Therefore, system resistance can be acceptably modeled by only taking into account the cell-patch-site resistance. The cell-patch site can be modeled as a conductor with a resistivity matching that of the $\mathrm{KCl}$ solution. This model is given by

$$
R=\frac{\rho l}{A}
$$

with resistance $R$, resistivity of the $\mathrm{KCl}$ solution $\rho$, cross-sectional area of the conductor $A$ (i.e. the area of the aperture), length of the conductor $l$ (i.e. the thickness of the membrane).

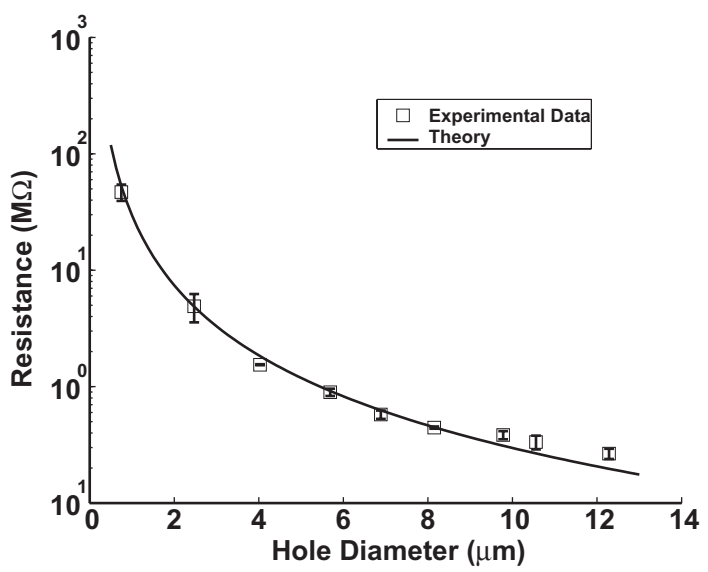

Fig. 10. Modeled cell-patch-site resistance and measurements. 
The impedance spectra and cell-patch-site diameter for a total of 27 thermally oxidized substrates were measured. Fig. 10 shows good agreement between the average resistance of nine different cell-patch-site diameters $(0.8,2.5,4.0,5.7,6.9,8.2,9.8,10.6$, and $12.3 \mu \mathrm{m})$ and the modeled cell-patch site resistance. The data points in the plot indicate the average of resistance measurements made from three different substrates while the error bars indicate the standard deviation.

The model for the capacitance of the substrate is given by

$$
\text { (2) } C=\left(\frac{1}{C_{F}}+\frac{1}{C_{B}}\right)^{-1}=\frac{\varepsilon_{r} \varepsilon_{o} A_{F} A_{B} d_{F} d_{B}}{A_{F} d_{B}+A_{B} d_{F}}
$$

with total capacitance $C$, front-side and back-side capacitances $C_{F}$ and $C_{B}$ respectively, relative permeability of the dielectric material $\varepsilon_{r}$, permeability of free space $\varepsilon_{o}$, the area of fluid contact, $A$ and thickness of the dielectric layer $d$ on the frontside $(F)$ and backside $(B)$ respectively. Equation (2) models the two capacitors formed by the conducting parallel plates of $\mathrm{KCl}$ solution and the silicon substrate separated by the silicon dioxide dielectric. Fig. 11 shows good agreement of the experimental data with the theoretical model and indicates that a small fluid-contact area is critical to achieving low-noise recordings. The data points in the plot indicate the average of capacitance measurements made from three different substrates while the error bars indicate the standard deviation.

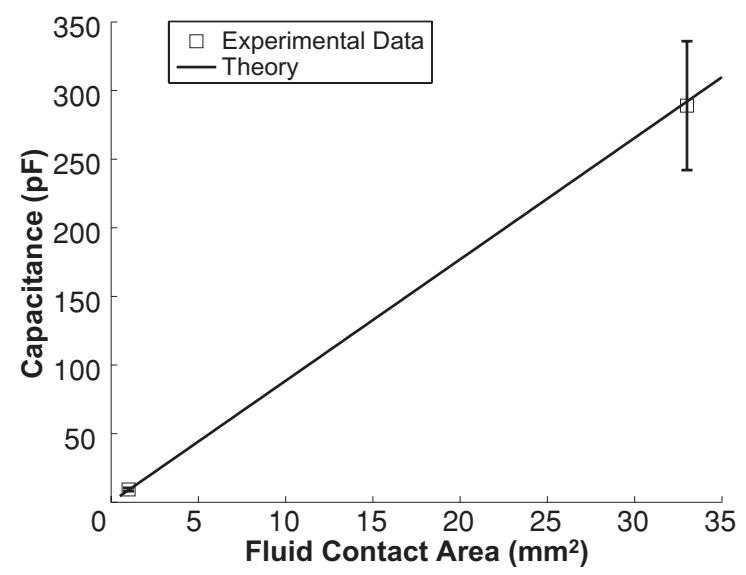

Fig. 11. Modeled substrate capacitance and measurements.

\section{Microfluidic Properties}

In operation, the fluidic system must be able to perform several tasks involving cell handling and fluid switching. For cell handling, the system must be able to drive a suspension of cells past the cell-patch aperture and trap a single cell from the suspension onto the aperture without damaging it. Once a cell is trapped on the patch site, the fluidic system must be able to switch fluids flowing past the patched cell in order to test the response of the cell to different test solutions. Qualitative tests have been made in order to test both cell trapping and fluid exchange properties of the microfluidic components of the system.

For the cell trapping tests, HEK cells are removed from cellculture flasks and suspended in standard Hank's balanced salt solution (HBSS). The cells are driven from the cell-inlet port, past the cell-patch aperture via a pressure differential with the waste port. In order to trap a cell onto a patch aperture, a pressure deferential is applied between the cell-inlet port and the backside port so that there is a net flow of fluid through the cell-patch aperture. When a cell is trapped, the pressure differential across the

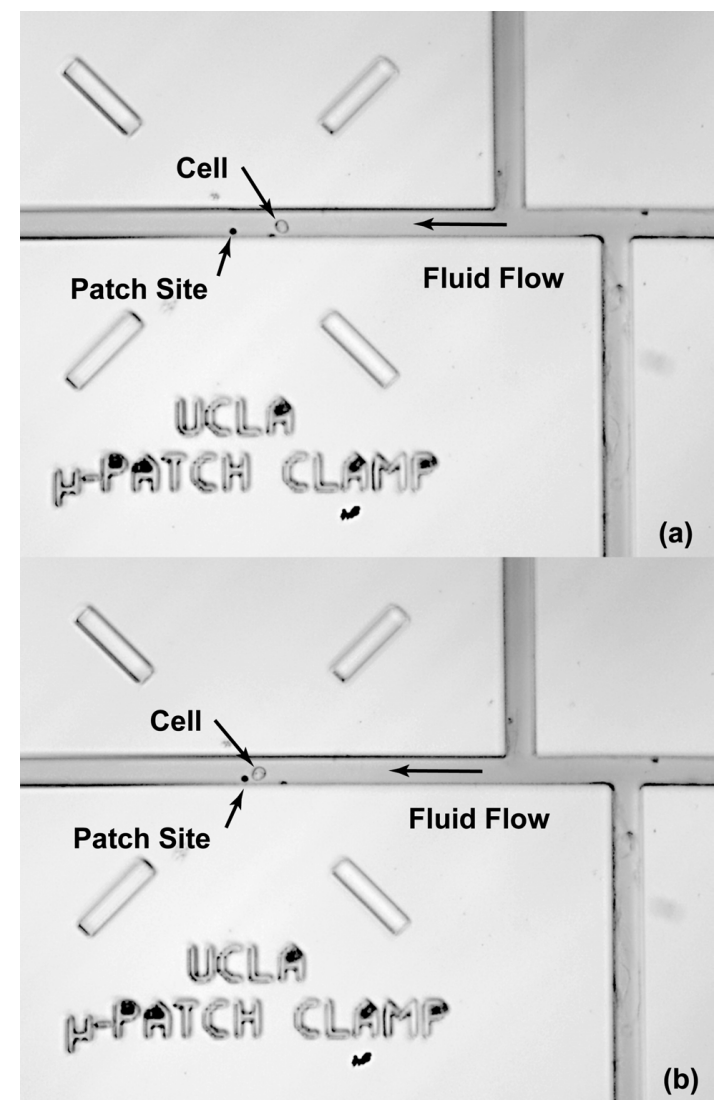

Fig. 12. Photograph of cell in microfluidic channel that is flowing toward a cell-patch site.

cell-patch aperture should be no more than $4 \mathrm{kPa}$, because a higher pressure could result in cell rupture before seal formation [21]. Using this method HEK cells have been successfully driven through the fluidic system and trapped on a cell-patch aperture (Fig. 12,13). Once a cell has been successfully trapped on a cellpatch aperture and a gigahom seal is made and the effect of ionchannel gating compounds can be tested by flowing an alternating sequence of buffer and ion-gating compounds past the cell. In order to qualitatively test the switching characteristics of the fluidic system, dyes representing ion-channel-gating compounds were driven through the system simulating extracellular fluid exchange (Fig. 14).

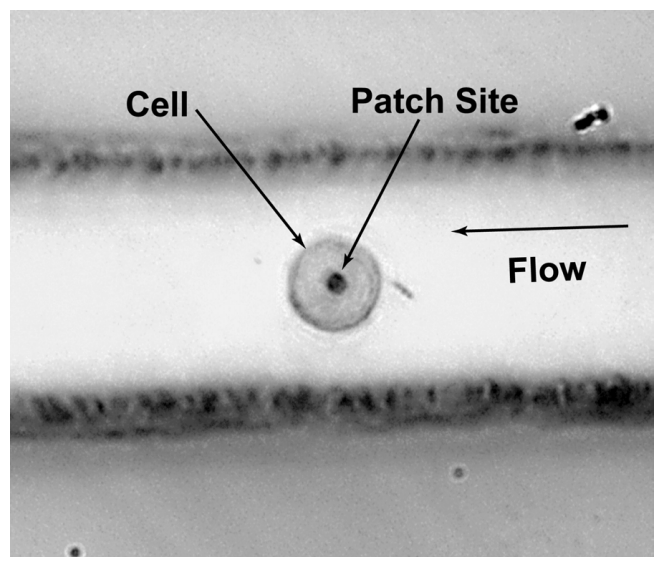

Fig. 13. Photograph of a cell in a microfluidic channel that has been trapped onto a cell-patch site. 


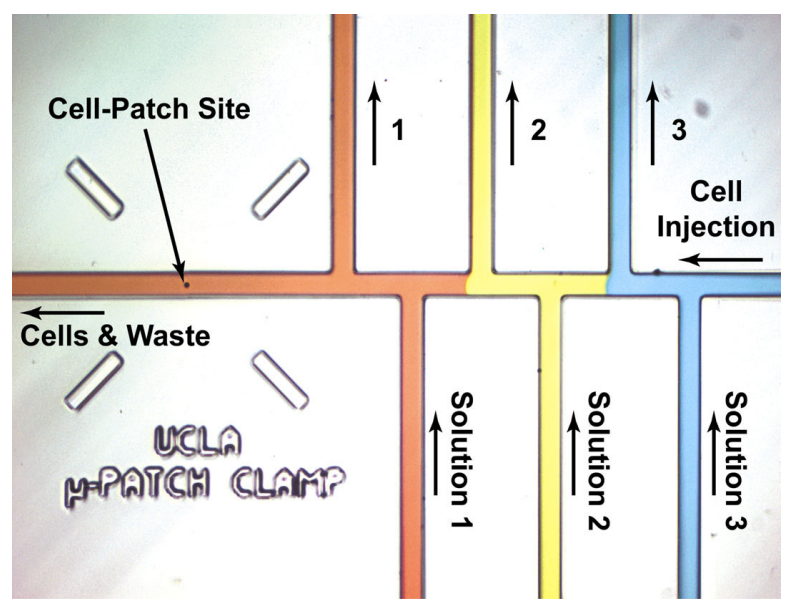

Fig. 14. Higher magnification photograph of the microfluidic system shown in Fig. 4. Three solutions are being passed through the microfluidic system to illustrate the formation of sample plugs.

\section{Sealing}

The formation of a gigaohm seal between the cell-patch aperture and the cell membrane is an essential component of patchclamp measurements. Without this seal, ions can leak between the membrane and patch aperture resulting in measured current that does not pass through ion channels and therefore contributes to error in the desired measurement. Currently, we are unable to form gigaohm seals with the microfabricated cell-patch apertures. This issue is currently the critical-path item limiting progress in the overall system. The primary reasons for poor seal formation in the microfabricated patch-clamp chips are hypothesized to be (1) fouling of the surface during microfabrication, (2) inappropriate surface roughness, (3) incorrect geometry of the patch aperture, or (4) fouling of the surface during cell capture. Experiments are currently underway to understand the dependence of these problems on sealing performance.

\section{CONCLUSIONS}

Specifically-engineered patch-clamp systems present an opportunity to simplify currently difficult-to-perform electrophysiology experiments. The first steps toward an automated single-cell dose-response-curve-measurement system have been made. To date, we have fabricated substrates containing cell-patch sites, fabricated PDMS microfluidic components, and integrated the two. The geometric and electrical properties of the silicon cell-patch substrates have been investigated and found to be suitable for patch clamping. Qualitative tests have been undertaken with the microfluidic components of the system to test cell handling and fluid switching. Although cells can be captured and different solutions can be controlled, gigaohm seal are elusive. Once gigaohm seals are reliably achieved, our patch-clamp system will hold the promise of parallelism, microfluidic manipulation, uniformity, optical access, and automation that is currently impossible to obtain with traditional patch-clamp systems.

\section{ACKNOWLEGEMENTS}

Supported by Center for Cell Mimetic Space Exploration (CMISE), a NASA University Research, Engineering and Technology Institute (URETI), under award number \#NCC 21364. Travel support has been generously provided by the Transducers Research Foundation and by the DARPA MEMS and DARPA BioFlips programs. We would also like to acknowledge the support of Prof. Montemagno's laboratory for their assistance with cell culturing and the intellectual collaboration of our colleagues in the UCLA Neuroengineering Program.

\section{REFERENCES}

[1] D. Purves, G. Augustine, D. Fitzpatrick, L. Katz, A. LaMantia, and J. McNamara, Eds. Neuroscience, Sinauer Associates. Massachusetts, 1997.

[2] Neher, E., and Sakmann, B., 1976, Single-channel currents recorded from membranes of denervated from muscle fibers, Nature 260:799-802.

[3] R. Pantoja, D. Sigg, R. Blunck, F. Bezanilla, and J. R. Heath, "Bilayer reconstitution of voltage-dependent ion channels using a microfabricated silicon chip," Biophys. J., vol. 81, pp. 2389-2394, 2001.

[4] C. Schmidt, M. Mayer, and J. Vogel, "A chip-based biosensor for the functional analysis of single ion channels," Angew. Chem. Int., vol. 39, pp. 3137-3140, 2000.

[5] N. Fertig, C. Meyer, R. H. Blick, and J. Behrends, "A microstructured chip electrode for low noise single channel recording," Biophys. J., vol. 80, pp. 337a, 2001.

[6] K. Cheung, T. Kubow, and L. P. Lee, "Individually addressable planar patch clamp array," 2nd Annual International IEEE-EMBS Special Topic Conference on Microtechnologies in Medicine and Biology, pp. 71-5, 2002.

[7] K. G. Klemic, J. F. Klemic, M. A. Reed, and F. J. Sigworth, "Micromolded PDMS planar electrode allows patch clamp electrical recordings from cells," Biosensors \& Bioelectronics, vol. 17, pp. 597-604, 2002.

[8] PatchXpress, Axon Instruments, www.axon.com.

[9] IonWorksHT, Molecular Devices,

www.moleculardevices.com

[10] Nanion Technologies, www.nanion.de.

[11] Sophion Biosciences, www.sophion.dk..

[12] B. Matthews and J. Judy, "Characterization of a Micromachined Planar Patch Clamp for Cellular Electrophysiology," IEEE-EMBS International Conference on Neural Engineering, pp. 648-651, 2003.

[13] Clariant Ltd., Data sheet: AZ5200 series, Mutteuz, Switzerland, April, 2000.

[14] Harrison, D. J., Fan, Z., and Seiler, K., "Integrated Electrophoresis Systems for Biochemical Analysis," Proceedings of the 1994 Solid-State Sensor and Actuator Workshop, Hilton Head Island, SC, June 13-16, 1994, pp. 21-24.

[15] J. McDonald, D Duff, J. Anderson, D. Chiu, H. Wu, O. Schueller, G. Whitesides, "Fabrication of microfluidic systems in poly(dimethylsiloxane)," Electrophoresis, vol. 21 pp. 27-40, 2000.

[16] Micro Chem, Data sheet: NANO SU-8 2000 Negative Tone Photoresist Formlations 2002-2025, Newton, MA, Febuary, 2002.

[17] C. H. Lin, G. B. Lee, B. W. Chang, and G. L. Chang, "A new fabrication process for ultra-thick microfluidic microstructures utilizing SU-8 photoresist," J. Micromech Microeng, vol 12 pp. 590-597, 2002.

[18] K. Hosokawa, K. Hanada, and R. Maeda, "A polydimethylsiloxane (PDMS) deformable diffraction grating for monitoring of local pressure in microfluidic devices," $J$. Micromech, Microeng, vol. 12, pp.1-6, 2002.

[19] B. Sakmann, and E. Neher, Eds., Single-Channel Recording, Plenum Press, New York, 1983.

[20] R. E. Thompson, M. Lindau, and W. W. Webb, "Robust, high-resolution whole cell patch-clamp capacitance measurements using square wave stimulation," Biophysical Journal, vol. 81, no. 2, p. 937-948, 2001.

[21] Z. Ahang, M. A. Ferenczi, and C. R. Thoma, "A micromanipulation technique with a theoretical cell model for determining mechanical properties of single mammalian cells," Chemical Engineering Science, vol. 47, no. 6 p.1347-1354, 1992. 